\title{
Enhancing Virtual Learning Spaces: the impact of the Gaming Analytics
}

\author{
Anastasios Karakostas, Anastasios Maronidis, Dimitrios Ververidis, \\ Efstathios Nikolaidis, Anastasios Papazoglou - Chalikias, Spiros \\ Nikolopoulos, Ioannis Kompatsiaris \\ Information Technologies Institute, Centre for Research and Technology Hellas \\ Corresponding author: Anastasios Karakostas E-mail: akarakos@iti.gr
}

\begin{abstract}
Online virtual labs have been important to educational practice by providing students with distance courses that otherwise would be difficult to be offered. However, the majority of them cannot be easily applied to different courses or pedagogical approaches. In order to overcome this, we propose a high-level, easy-to-use authoring tool that will allow building course-independent highstandard virtual labs. This solution is based on learning and gaming analytics. In the gaming industry, there have been developed strong game analytics methods and tools, which could be easily transferred into the learning domain. Game analytics monitor the users' activity; model their current behavior through the use of shallow analytics and predict the future behavior of the users through the use of deep analytics. We propose that both of these approaches combined with visualization methodologies will offer insights on what features are important and what functionalities users expect to find in a virtual lab.
\end{abstract}

\section{Introduction and motivation}

Information and Communication Technologies (ICT) has enabled the spread of personalized learning across the largest part of community. One of the advantages of technology-enhanced learning is the ability to offer automated methods, which are easy to be accessed for example through the web. In this context, recently there have been developed a number of virtual labs emulating real lab environments, where users can accomplish a number of learning tasks and conduct various experiments with no cost and risk. However, the big challenge of such labs is to find effective ways to boost the experience factor in order to motivate the engagement of students with the learning system and prevent them from churning out. In this context, the overarching goal of our framework is to enhance the design of virtual labs leading to optimal personalized learning processes. Towards this end, the framework consists of an authoring environment, which equipped with data analytics methods and visualization tools that have been developed and 
reached maturity in the gaming industry and is suitable for iteratively evolving the design of virtual labs and for dynamically adapting the learning content to the users of the virtual labs.

This environment operates in a high level requiring minimal programming skills, so as to be a powerful tool in the hands of every designer (e.g., tutor) who will be able to construct a lab that meets the requirements of the users. For the optimization of a virtual lab, it is imperative that the designer has access to data and information about learners and their contexts. In our framework we make use of available data logs encoding user activities that have been acquired from already existing virtual labs. Moreover, apart from these data, it is also needed to keep track of the activity of users in new labs so as to keep all crucial information updated providing feedback to the process of enhancing virtual labs. Understanding the data obtained from both existing and new virtual labs, is possible to be reached by means of Learning Analytics (LA).

Learning analytics is a useful tool that allows for deciphering the trails that learners leave during their engagement with the learning system. They can have a strong impact on the learning process (e.g., for educators to identify at-risk learners, or for learners to reflect on their learning process), but also allow for more informed decisions on the institutional, national and international level (e.g., for determining the value generated by faculty activity, or for implementing what-if scenarios using predictive behavior models). Given the volatile nature of the data and information acquired during the learning process, analytics must be conducted at a pace that can instantly inform decision-making in both aspects of feedback to learner and adjustment of the learning system. This means obtaining real-time or near real-time answers from data by having automated systems that continually update user profiles, contextual information, etc.

Towards this end, we anticipate that the gaming industry and its knowledge resources can give the answer. Indeed, due to the recent intense activity in games, there have recently been developed strong game analytics methods and tools, which could be easily and seamlessly transferred into the learning domain. Game analytics have the potential to contribute in two ways. First by monitoring the activity of the users and modeling their current behavior through the use of shallow analytics (simple statistics on tracked data). Second by predicting in a reliable manner the future behavior of the users through the use of deep analytics (outcome of the application of machine learning algorithms). Both of these approaches combined with state-of-the-art visualization methodologies will offer insights on what features are important and what functionalities users expect to find in a virtual lab. These insights will allow for a) optimizing the design and implementation of a virtual lab and $b$ ) enhancing the learning process in a virtual lab by offering personalized learning content.

Summarizing the above, the overall concept of our solution is based on iterating the process of improving virtual labs through a pipeline that i) starts from the current version of a lab, ii) collects shallow analytics extracted from user behavioral data, iii) digs deeper into the obtained analytics using machine learning 
methods, iv) integrates the obtained information under the authoring tool, v) employs the authoring tool to build an improved version of the virtual lab and finally vi) iterates the above process.

\section{Background and literature review}

\section{Learning analytics \& Game analytics}

Learning analytics focus on assessing the learning process, and thus are useful on a course level (for e.g. identifying the learning trails and engagement of individual learners) as well as on a department level (for e.g. predicting dropout rates and clustering learners) (Siemens \& Long, 2011). The critical dimensions of learning analytics are the stakeholders (e.g. learners or tutors), the objectives (e.g. reflection), the data (e.g. which measures are important, whether a learner's history will be considered), the instruments (e.g. data visualization or statistical analysis), the external constraints (privacy, ethics, etc.), and the internal limitations (e.g. whether the analytics can be interpretable by the stakeholders) (Greller \& Drachsler, 2012). Choosing along each of these dimensions can severely affect the quality, accuracy but also the usefulness of the analytics. As indicative examples along the dimensions of data and instruments, Marist College used demographic data (such as the learner's age and gender), general scores (such as the learner's SAT score) and LMS-specific data (such as the number of times a learner accessed a Lesson section) as input data, in order to assess a learner's academic risk (output data) (Lauria et al., 2013). Another example is SICKO , a web-based educational game where positive and negative feedback is provided by a virtual doctor; the included Surgery Mode mini-game has the players determine the fate of a virtual patient by answering multiple-choice questions (Shieh et al., 2012), (Tsui et al., 2014). The game's collected data include an action log of the learner's answers to the multiple-choice questions, along with the elapsed time between answers and score progression. Since the score was a direct indication of the number of right and wrong questions (and rewards to score is scaled to the difficulty of the question), the score could be used directly as output data for assessing the success of students. Moreover, by visualizing the distribution of scores in a figure (with the y-axis being the number of students achieving this score), the stakeholder (in this case the game designers) could assess whether there were many learners who underperformed - in which case the game may have required a re-design.

Of particular importance our solution is the data and instruments dimensions, i.e. which data from a learner's interaction with the LMS can or should be collected, and which methods are used to inform the stakeholder of this interaction. In our framework, the stakeholders are the educators as well as the designers of the 
new virtual labs (these roles may overlap). Therefore, importance is also placed on which data could be valuable to these stakeholders, and how to visualize it in order to best inform them of the interaction process of learners. For instance, educators using the learning analytics to observe how a large number of learners interact with the LMS (Learning Management System) are likely to be interested in a single metric denoting number of problem cases with insufficient learning which are likely to dropout. In such cases, game analytics can be employed to derive a likelihood of dropout; similar methods are used to predict when players stop playing a particular game, i.e churn prediction (Runge et al., 2014). On the other hand, a designer of the new virtual lab will not find such a metric sufficient; instead, more informative data include session times (long or short session times may indicate problems) or a visualization of which elements of the interface were most clicked at (for identifying interface design problems where some buttons were missed). Game analytics for identifying how users engage with game development tools (Liapis et al., 2014) can be transferred to such visualization and assessment tasks.

\section{Visualization}

The almost ubiquitous use of the computer (in any of its forms, including the personal computer, mobile devices, surveillance systems) in all aspects of everyday life - not least of which in education - necessitates an efficient way to present this data to different stakeholders. Similar to learning or game analytics, which identify key performance indicators and filter the vast amounts of data into comprehensible numbers, the visualization of data manages to distill information in an intuitive and easy to grasp imagistic representation. The core idea behind data visualization is that a diagram, through its use, serves as a vehicle of cognitive processes, embodying the various aspects of the problem. The viewer's (e.g. a stakeholder's) mind is extended (Clark, 1998) onto the diagram and reasoning proceeds through structural (rather than semantic or syntactical) entailment. One therefore thinks through the diagram rather than its use as a simple image. Moreover, the possibilities one sees for constructing, altering or transforming a given diagram are part of one's comprehension of the diagram itself; the functions of the diagram both on the semantic and pragmatic level are determined in part by these possibilities (Sloman, 2002). Therefore the visualization of data serves three purposes to a user (or stakeholder): (a) to promptly understand the current state of a particular domain based on the key performance indexes that interest them, (b) to reason about the data, detect re-occurring patterns and predict future or unseen data by projecting the current data into unknown problems, (c) to envision ways of changing the diagrams themselves and the necessary steps taken to achieve that. Using more concrete examples, the first purpose is achieved when observing e.g. a summary of the hours spent by each group of learners (class) on a specific virtual lab, the second purpose is achieved when observing e.g. a timeline of the correct re- 
sponses of one learner which shows an increase in correct responses from the beginning of the semester until now and the educator can extrapolate a similar trend occurring until the end of the semester. Finally, the third purpose can be achieved by observing e.g. how students are automatically clustered based on their engagement time with the virtual lab, showing a cluster of students which have low participation and low correct answers; the educator can attempt to indirectly affect this diagram by attempting to reinforce the learners' engagement with the tool and improve their rate of correct answers by either in-class seminars or by adapting the virtual lab directly to provide more help to at-risk learners.

Commercial games often collect a broad range, large volume of data from their players; this is facilitated by the modern advances in network speeds and bandwidths, but also by the more robust ways that the industry has adopted to reason about and visualize such data. The data is necessary for deriving game analytics — including player satisfaction, game balance, and many others - which in turn act as the key performance indicators and need to be communicated back to the relevant stakeholders. For visualizing this data, several methods have been developed by the game industry (and other domains): (a) displaying descriptive statistics numerically (e.g. in a table), which can be beneficial as they are easy to read and maintain the fine details (e.g. numerical precision), (b) displaying trends over time (e.g. values of key performance indicators on a timeline) which allows stakeholders to predict future outcomes based on current and past trends, (c) displaying heatmaps which can easily identify user's spatial navigation and highlight elements in level design or interface design, (d) display groupings of players clustered based on certain shared (actual or predicted) attributes. These visualizations do not need to exclusively consider the game's designers (or producers, in case of financial figures of the game) as the only stakeholders: in many massive multiplayer online games or competitive games, the end-users (players) largely benefit from such visualizations to adjust their strategies when facing other players: this is done by e.g. observing which hero classes are trending currently based on how often they are picked by other players, visualized both as quantitative descriptive statistics and as a timeline. Heatmaps "are basically frequency maps - they show how often a particular event has occurred - traditionally a player defeat event on a fine-meshed grid, overlain a map of the game level in question" (Drachen \& Schubert, 2013). Beyond player defeat, heatmaps have often been used to visualize how players traverse a level or interact with elements of the user interface. Finally, clustering can be used to group similar game elements (e.g. game items, players, questionnaire responses) based on real-world gameplay analytics collected about them in a way that elements in the same cluster are more similar with each other than with those in other clusters: for instance, clusters can be visualized in order to show the number and ratio of distinct types of players by interpreting a broad range of their gameplay.

When moving from games to learning environments, the goals of data visualization do not particularly change: treating either learners or educators as the primary stakeholders, visualizing data in more or less concise - yet intuitive - 
ways can provide an overview of the learner's progress and can be used to predict (by human viewers) the outcomes of future tasks. Our approach explores how the best practices of data visualization used in games can be used to provide information to both stakeholders: learners and educators. For learners, data visualization of their progress (e.g. of their correct answers) over time, possibly compared with the trends (average values) of other learners can motivate them to continue using the virtual lab (averting churn). For educators, who take a similar role to the game designer in game industry terms, the full spectrum of data visualization can be used very effectively. For educators, displaying descriptive statistics numerically can provide them with enough information (at a glance) during the progress of a lecture to intervene directly, while visualizations of progress over time can allow them to predict the outcomes of future exercises (since they have an overview of the difficulty of the virtual labs they designed) and take precautions by editing upcoming labs. When designing a new virtual lab, on the other hand, the educator can make use of heatmaps of learners from previous virtual labs' interfaces to improve usability of the interface in future iterations.

\section{Research motivation}

Work on coupling game analytics with learning analytics we will advance the research in both fields, as (a) expertise in deep game analytics which can predict future trends can be transferred directly into predicting learning outcomes of learners engaging in virtual labs (using learning analytics as input or predicted output); (b) the translation of play metrics in educational settings will provide significant insights going forward in games which are used for educational purposes, such as SICKO (Tsui et al., 2014); (c) application and testing of deep game analytics in educational software can result in new algorithms which can be used for any type of data, including game industry applications, personalized playlists and product suggestions etc.

Moreover, during the course of our framework, the research and implementation of data visualization practices in the realm of educational software will advance the state of the art in this domain in several ways: (a) new methods of visualizing the findings of deep game analytics (or deep learning analytics) for nonexpert users in machine learning and artificial intelligence will open up the user base which can benefit from such methods; (b) new findings on usability of interfaces using sophisticated heatmaps, play-traces, and quantitative results can be transferred back to the game industry (where the focus is less on interface elements and more on spatial navigation); (c) can study the impact of thinking through diagrams in educators and learners (possibly comparing between the two) from a human- computer interaction perspective, increasing the knowledge going forward for the needs and priorities of these types of stakeholders. 


\section{Proposed approach}

Our framework is aimed at providing education with all those technologies necessary to enhance virtual labs from the perspective of both a teacher and a student. Towards this direction, we adopt a data-driven approach by transferring to education well-defined and mature data analytics technologies, which have shown extremely high performance in the gaming industry. The use of a data-driven approach is motivated by the continuously increasing need to apply learning analytics to massive, varied and time-sensitive data, acquired from the engagement of users with virtual learning environments. Learning analytics focus on assessing the learning process, and thus are useful on a course level, e.g. for identifying the learning trails and engagement of individual learners, as well as on a department level, e.g. for predicting dropout rates and clustering learners (Long \& Siemens, 2011). In this context, the critical dimensions are

- the data generated by the learners' activities;

- the analytics tools that are used for collecting, aggregating, interpreting, visualizing and understanding these data; and

- an authoring environment that integrates the above in the process of improving virtual labs. In the following we give a description of these critical dimensions.

\section{Insert Fig1}

Fig.1. Concept diagram.

The virtual labs authoring tool consists of three components, namely a) the front-end that shows simple visualizations to the educators in order to author games, b) the back-end that shows advanced visualizations to administrators of the website and how to define new Game Project, Scenes, and Assets3D taxonomies, and c) the assembler-compiler that combines all Game Scenes, Assets and Settings into a Unity3D project and compiles it into a game. These components will be outlined next.

\section{Front-end interface}

The front-end interface is a user-friendly Graphical User Interface (GUI) that allows the management of Game Projects, Scenes, and 3D Assets, effectively targeting novice users, that have limited or no knowledge of game authoring processes.

Firstly, after successfully logging in to the website, the user enters the Game Project Manager screen that offers functionality to create a new game, and edit or delete an existing game. Next, the Scenes Manager screen is shown, in which an existing scene can be edited or deleted, and a new scene can also be created. There is a link to the $3 D$ Asset Manager screen and if desired, the user can compile the 
full game from this screen. If the user selects to edit a scene, then the Scene Editor screen appears, with contents that depend on the type of scene. For 2D scenes a form is shown that the user can submit to change its data. For 3D scenes a fully functional $3 \mathrm{D}$ editor is show so that the user can spatially manipulate and arrange $3 \mathrm{D}$ assets in a plane. All screens are described in greater detail in the subsequent sections that follow.

\section{Insert Fig2}

Fig. 2. Overall organization of the front-end GUI.

\section{Game Project Manager}

An implementation of the Game Project Manager is shown in Figure 3. In this screen, the educator can create a new game project and delete or edit an existing one. To access a preexisting project, the educator must click on one of the list entries at the left Projects section. There is also a delete button that creates a warning popup window to make sure that the educator really wants to delete this project. A new game project can be created by entering the title of the game project and by selecting its type. When clicking on the CREATE button, a new game is successfully created and the educator is transported to the Scenes Manager screen.

\section{Insert Fig3}

Fig. 3. Implementation of the Game Project Manager

\section{Scenes Manager}

As shown in Figure 4, in this interface each scene of the game is represented as a card. Each card has a thumbnail of the scene that also serves as a link to the scene editor, a scene title, a description and two buttons for the edit and delete scene functionalities. There are some scenes that the game project manager creates by default. These scenes are required and cannot be deleted so the delete functionality is disabled, although all scenes that are created by the educator are deletable.

In this screen there is also the 'Compile Game' button that when clicked initiates the compilation process of the whole game. A new screen appears that allows the educator to compile the game in various formats such as WebGL, Windows, Mac, or Linux. Upon successful compilation a link is provided to download the game in zip format. For WebGL games, a second link also appears to play the game in the web browser.

By clicking on the 'Add New Scene' button, a new section expands that enables the creation of a new scene by filling in the necessary information. This infor- 
mation includes a title, a description and an image that serves as a scene thumbnail.

\section{Insert Fig4}

Fig. 4. Implementation of the scenes manager.

\section{Scene Editor}

When the scene that is being edited is two-dimensional, the 2D scene editor launches that is in fact a form with fields that vary according to the scene. If the scene that is being edited is three-dimensional then the $3 \mathrm{D}$ scene editor launches. We have developed a web-based 3D Editor to enable modifications of a scene through a web-browser. In order to achieve this fast, we used the three.js ${ }^{1}$ library that allows to develop 3D graphic elements using HTML5 and WebGL through high level commands. Three.js allows saving a scene in the JSON format where we have standardized our own custom structure that serves the need of converting the scene setup to the Unity3D scene format. A screenshot of the 3D editor can be seen in Figure 5.

\section{Insert Fig5}

Fig. 5. A scene editor for the web using Three.js.

The 3D Editor screen consists of three basic parts, namely the 3D view of the Scene where the user can manipulate 3D objects on a plane, the left panel that features controls and editable parameters of each selected object, and a right panel where all available 3D assets are listed and can be dropped inside the 3D plane, edited or deleted. There is also a search function for the scenarios where one Game Project has numerous 3D assets.

The main functionality of the 3D editor is to allow an educator to drag and drop 3D assets on the 3D plane. This action adds an instance of the 3D asset to the scene. Multiple instances of the same asset can exist in the scene, i.e. multiple wind turbines. The educator can edit the rotation, position, and scale of an instance either through GUI controls (gizmos) or by entering numerical values for a more accurate result in the left panel. Other functionalities supported are typical 3D editing functionalities such as a) view the scene either in 3rd person view or 1st person view; b) orbit, pan, or zoom to an object for a better angle view; and c) select object with raycasting (click on 3D items).

The 3D editor, apart from the editing functionalities, can convert a three.js scene into a JSON file. A three.js scene is comprised of objects in the browser's memory that are structured in a tree like format with parameters such as object

\footnotetext{
${ }^{1}$ http://threejs.org
} 
name, translation, rotation and scale. We have developed a JSON converter function that stores these parameters inside a JSON file following a protocol. We have defined our own protocol as for the time being there is no standard format for saving three.js $3 \mathrm{D}$ scenes. If the educator re-opens the $3 \mathrm{D}$ editor for a particular scene, then the JSON file is loaded and the three.js scene is recreated exactly like it was saved the last time it was edited. We decided to use the JSON format instead of the Unity3D YAML scene format because it is more compatible with web technologies such as three.js. Only when the game is compiled, the JSON scene is converted into a Unity3D YAML scene.

In a recent version of the plugin, game analytics are embodied in a tab next to $3 \mathrm{D}$ editor as shown in Figure 6. The analytics contain information regarding the entities such as the game, the scenes, and the assets. The parameters for each entity regard the frequency of use, the duration of use, game score statistics etc.

\section{Insert Fig6}

Fig. 6. Analytics are displayed in a tab next to scene 3D editor.

\section{D Asset Manager}

The educator can upload a new 3D asset using the "Add new Asset" button, which is only available for senior educators, and is accessible from the Scenes Manager or the 3D editor screens. The 3D Asset Manager screen is shown in Figure 7. Each 3D Asset has various fields such as files for 3D representation (obj, mtl, jpg texture) and various fields for its parameters (e.g. power consumption mean and variance). The category of the asset affects the kind of fields of the asset. Further information about the categories of the assets will be described in Section 3 for the certain use case.

Analytically, the steps to create a new 3D asset are a) select the category of the asset based on the type of the current game project, b) write the title and an optional description, c) upload the 3D representation files that include an mtl (material) file, an obj (mesh) file, and a jpg texture file, and d) set the asset fields based on its category. The $3 \mathrm{D}$ model is rendered in a panel and the user can save a snapshot of the 3D model to be used as an icon during the 3D Editor.

A 3D asset can be edited, when clicking on the 'Edit' button from the 3D Editor (Scene Editor) screen. All information of a 3D asset can be edited except from its category.

Insert Fig7

Fig. 7. The 3D Asset Manager for the energy "Consumer" asset of the "Energy" Game Type. 


\section{How a generated game looks like}

The games contain by default certain 2D scenes, which allow the basic functionalities of games with GUI elements. These 2D scenes are outlined in the following:

Main Menu - It is the central point of the game where the learner can select what to do next as shown in next Figure. The title, e.g. "Renewable Energy VR Lab" and the image below the title are editable from the virtual labs authoring tool. All the other GUI elements are fixed. An option is provided to hide Login, Settings and Help if the educator does not wish to have these buttons available.

\section{Insert Fig8}

Fig. 8.: Main menu scene

Login - Button loads a Scene where the input fields for the learner name, the surname, and the school can be found. An example is shown in Figure 3.2. The information is encrypted before transmission into a unique identifier that can not be inversed, i.e. there it is not possible to extract a learner's name from its encrypted identifier. This scene is not editable from the authoring tool.

Insert Fig9

Fig. 9.: Login scene

Settings - Button loads a scene that provides controls for changing screen size and details level as shown in Figure 3.3. This screen is useful in low-end devices that should have a low resolution and details level in order for the game to be played smoothly. This scene is not editable from the authoring tool.

Play - Button loads a scene named as "Scene Selector" that allows the user to select an Educational Scene to play among several choices. An example is shown in Figure 3.6. This scene is automatically generated from the authoring tool based on how many Educational Scenes are available. The title, description, and image of the scene are those fed as input during the creation of each scene. The title "Select a Scene" is editable. Next, the Educational Scenes are described.

Innsert Fig. 10

Fig. 10. Scene Selector scene allows to select a "level".

Educational Scenes - These are the energy production-consumption simulation scenes that contain the main interaction for achieving the learning objective. An example is shown in Figure 11. Here the energy consumers are the buildings 
which are colored as red indicating that they are underpowered. Above each building, a billboard shows the mean and the variance of the consumption of each building. The circles in the terrain indicate candidate positions for inserting a wind turbine. On hovering above each cycle, information of the candidate turbine is shown. On clicking a cycle, a turbine is built over it. Depending on the size of the turbine rotor the nearby candidate positions are destroyed automatically in a range of 1.5 times of the rotor size. When hovering on each turbine a billboard over the turbine shows the characteristics of the turbine as well as the current output for the current wind speed. On bottom-left, the current state of the game is shown. This state consists of the following metrics: the total energy produced so far, the money earned, the required power, the generated power, and the wind (current, mean, variance). The simulation lasts 6 minutes in real time that corresponds to 24 hours. A turbine can be damaged after some time, and the learner can click on it to repair it with some cost. The interactions allowed by the learner will be described in Section 3.3. Upon finishing the simulation the Reward scene is shown which is described next.

Insert Fig11

Fig. 11. The simulation of energy production-consumption constitute the educational scenes.

Reward Scene - As shown in Figure 3.8, contains the final score and an overall evaluation for the simulation session. The learner can see his or her score as the money earned, the energy produced and the balance of among overpower, correct power, and under-power time.

\section{What the educator can do with the Virtual labs authoring tool using the "Energy" lab template}

The "Energy" template can be used to generate an arbitrary number of games, with an arbitrary number of Educational Scenes, and with an arbitrary number of game objects per Educational Scene. In the following lines, we will describe which parameters the educator can change by using the vlabs authoring tool. Briefly, the vlabs authoring tool copes with the following requirements.

- Allow the learner to select an Educational Scene to play among several Educational Scenes. Each scene has its own pros and cons that should be explained to learner.

- The energy consumption per scene should be modifiable.

- The wind speed per scene should be modifiable.

- Wind energy turbines should have the following modifiable parameters

- Power Generation 
- Size

- Cost to buy

The educator can do the following actions in the first prototype of the vlabs authoring tool

[Action A] Create multiple Educational Scenes: Each of these scenes is a certain area where the wind energy generators can be placed.

[Action B] Insert a Terrain: A Terrain is a ground where turbines can be placed. This action is feasible by drag-n-drop an Asset3D to the scene. Figure 12 shows a scene with a terrain. Only one Terrain can be placed in a scene.

\section{Insert Fig12}

Fig. 12. Inserting a terrain with drag-n-drop from right toolbar.

The Terrain has the following fields that should be defined by the educator by pressing the "Edit" or "Create new Asset".

[Action C] Insert a Decorator: A Decorator is a game object that can improve the immersiveness such as "Archaeological site", "Power lines", "Trees", etc.. Their category, which should be selected when creating a new asset, is named as "Decorator". Decorators can be dragged-n-dropped an arbitrary number of times in the scene

[Action D] Insert a Consumer: A Consumer is a game object that consumes energy (e.g. a building). Several Consumers (block-of-flats, single houses, factories) will be available for drag-n-drop in the scene for multiple times. The total energy consumption is the sum of the consumption of all Consumers. A Consumer turns red if underpowered, blue if overpowered, and normal color if correctly powered.

[Action E] Insert a Producer: A Producer is a game object that generates energy (e.g. a Wind Turbine or a Solar Panel). Producers can be dragged-n-dropped several times in the game by the educator. When the game starts they do not appear but a marker is shown on the ground to indicate to the learner that in this place where a Producer can be built (Candidate position).

\section{Learner actions allowed in the produced games}

Here we provide a summary of the actions that learner can perform with respect to the learning objective.

[Action A] Select an area: to place the turbine among several choices where the pros and the cons are stated.

[Action B] Place turbines in candidate positions: A candidate position is shown with a marker 
[Action C] Turn off a turbine - The learner can turn-off a turbine by clicking on it when the power generation greater than the consumption.

[Action D] Repair a turbine - The learner can repair a turbine if the turbine outputs smoke by clicking on it.

[Action E] Change simulation speed - The learner can change the simulation speed using the top-middle dropdown button.

Other navigation and visualization features are :

- The learner can orbit-zoom-pan around the scene to see the turbines from all sides.

- The learner can view money earned, current energy productionconsumption, and current wind speed in the lower-left panel.

\section{Visualization of Deep and Shallow Analytics}

In order to analyze, model, and eventually visualize, learner behavior through shallow and deep analytics, we are implementing three separate technical solutions. The three solutions are interdependent and form a logical and operational stack, that can be deployed as one, and hence all three of them are described in this section.

The user-facing layer of the stack is the visualization service. This service displays observed and inferred information about the learners of a given virtual lab and informs the author of behaviors in the currently implemented version of the virtual lab as well as potential outcomes of changes to the virtual labs. The visualization service in turn receives its information from the shallow analytics service.

The shallow analytics service performs data aggregation, abstraction, and storage tasks, taking raw measurements gathered from the virtual labs and turning these into metrics that can be analyzed by the user. The shallow analytics service communicates with the deep analytics service that receives data, models the data, and transmits this back to the shallow analytics service which in turn stores the results and provides these to consumers i.e. the visualization service.

Insert Fig13

Figure 13: Overview of the visualization, shallow analytics, and deep analytics stack. Blue: services and their core functions; Yellow: necessary platforms; and Orange: used libraries and custom code. 


\section{Measured Data}

The raw data points as they were described in Section 4, Table 4.1, are converted into a number of metrics by the shallow analytics service. Depending on the use case these may be converted on-line in the user's browser or off-line and stored with the data set. All events are grouped by user and session and turned into list data structures with one list per user per session. Individual sessions are demarcated using the "launch" event described in Table 4.1. If no further events are received from the same user for an extensive amount of time, the session is considered concluded.

With each list representing a series of events for each session for each learner, it now becomes possible to leverage the other event types to evaluate the learner's travel path through the application, as well as calculating the five key metrics of interest, defined in previous deliverables. Further, combinations of these features constructed from expert knowledge, as well as the raw event data, may be transferred to the deep analytics service for treatment.

\section{Validation}

The general objectives of piloting and evaluation tasks are to conduct a series of small-scale test implementations of virtual labs, accompanied by the authoring, analytics and visualization tools, in the context of well-defined educational scenarios. This is done to evaluate the effectiveness of the developed technologies with respect to their ability to benefit educational organizations utilizing the our solution towards the optimization of virtual lab design and functionality. The evaluation is foreseen to provide quantitative and qualitative feedback on three separate conditions:

a. The process of using our authoring tool as a means for building virtual labs.

b. The offered analytics and visualization tools as means of support towards improving virtual labs.

c. The delivered virtual labs with respect to their effectiveness to meet the goals and expectations of both teachers and students in the learning process.

In particular, the process of using the authoring tool as a means for building virtual labs will be evaluated by teachers and e-learning experts as test users. Predefined tasks of gradual complexity will be given to them and feedback will be collected through various means and methods in order to evaluate the effectiveness, usability and functionality of the authoring tool as well the overall user experience. The evaluation outcomes will be utilized to develop, update and release an improved version of the tool. 
With respect to the support offered by the analytics and visualization tools in the process of improving virtual labs, this will be evaluated also by teachers and experts. Similarly, these tools' effectiveness, usability, functionality and user experience will be assessed towards their improvement and upgrade.

The delivered virtual labs and the learning content will be evaluated by both teachers and students as well as experts. The evaluation process will focus on measuring to which extent the virtual labs have achieved to motivate and to engage students in the learning process, and also to which extent their utilization in the classroom facilitated the teaching and learning objectives set or expected by teachers.

\section{Discussion}

Analytics, apart from the gaming industry, have also been used in many different contexts, e.g., healthcare, stock trading, web store customer analysis, retailing and marketing optimization, just to name a few. However, there are two main reasons that make game analytics special in the context of learning, which are described hereunder.

Coherence between gaming and learning: Gaming and learning are intertwined processes, both of which simultaneously appear in the early stages of a child's development and thereby they bear some fundamental similarities. Indeed, gaming and learning aggregate numerous similar traits (e.g., they stimulate fantasy, trigger curiosity, intrigue users by offering challenging goals, arouse enthusiasm, entice users by offering several types of rewards, foster creativity through the use of nonlinear approaches, etc.), which have permitted the fusion of these processes towards producing hybrid approaches like for instance "learning by playing" and "edutainment". Due to this inherent connection between gaming and learning, the users in both of these processes generate common types of data and therefore it is anticipated that data analytics methods that have been successfully applied to gaming have a strong potential to also be successfully transferred to learning. Based on the above principle, our solution will guarantee this smooth transfer by designating and using a set of learning-biased metrics for assessing the data collected from users interacting with virtual labs.

Strength of game analytics: Due to its popularity and the demanding requirements of the several stakeholders involved in it, the gaming industry has revolutionized the development and optimization of analytics. While conventional analytics have been built mainly to optimize the e-commerce experience, e.g. through naïve statistical approaches for measuring the recency, frequency and monetary value of a user, game analytics basically focus on making a game more engaging, e.g. through user behavior modeling, predictive analytics and visual analytics. Along these lines, there have been developed game analytics tools offering a variety of metrics (e.g., average session duration, performance metrics, player de- 
mographics, etc) that allow for adapting the game play so as to suit the style of different game player-individuals or segments. As such analytics have demonstrated tremendous accuracy in games, it is anticipated that they can be equally effective when transferred to learning

To demonstrate their effectiveness, the transferred technologies adopted by our solution will be rigorously tested and evaluated by real-users and in realistic small-scale learning scenarios. The goal of this process will be on the one hand to validate the integrity of the technologies developed throughout the project and on the other hand to verify that the developed solution matches the end-user expectations. Succeeding in both of these goals will imply the smooth transfer of game technologies to education.

\section{Conclusions}

As a term, learning analytics has only recently been introduced to describe the "measurement, collection, analysis and reporting of data about learners and their contexts, for purposes of understanding and optimizing learning and the environments in which it occurs" ${ }^{\text {. }}$. While still nascent, the field of learning analytics has received considerable attention. The primary reason for the interest in learning analytics is the vast and diverse data being collected by online Learning Management Systems (LMS); LMS themselves have also seen overwhelming adoption rates, with $99 \%$ of US colleges and universities using such systems. While adoption rates in Europe have been less pronounced in the past, recent years have seen a growing European interest in e-Learning, leading to growth in this sector ${ }^{3}$. Learning analytics are both an affordance and a necessity for the immediate future of learning institutions, since (a) data is now accessible in real-time (as it is produced), (b) computers possess the necessary computing power to process vast volumes of data, and (c) new types of data (e.g. via sensors or connected devices) become available ${ }^{4}$. Identifying and implementing appropriate learning analytics can have an important impact both on the learning process on the individual level (for educators to identify at-risk learners, or for learners to reflect on their learning process), but also allow for more informed decisions on the institutional, national and international level (for determining the value generated by faculty activity, or

${ }^{2} \mathrm{G}$. Siemens. Call for papers of the 1 st international conference on learning analytics \& knowledge. https://tekri.athabascau.ca/analytics/call-papers, 2010.

${ }^{3}$ E. Group. Increased e-learning investment in europe points to a market on the turn. http://edxusgroup.com/increased-e-learning-investment-in-europe-pointsto-a-market-on-the-turn/

${ }^{4}$ M. Mayer. Innovation at google: the physics of data. Presentation at the PARC forum, 2009. [Slides available online at http://www.slideshare.net/PARCInc/]. 
for implementing what-if scenarios using predictive models) (Long \& Siemens, 2011).

In this context and motivated by the expected impacts of the call, one of the main objectives of our framework is to identify which game analytics technologies could be migrated to education and explore effective ways to make a smooth and seamless transfer. As current virtual labs already use several types of analytics tools, it is important to detect and exploit the advantages that game analytics can offer against current technologies. As the optimization of such technologies is synonymous to impact maximization, keeping in-touch with state-of-the-art analytics is very crucial for the survival of businesses in the educational industry. For this purpose, the progress of performance and optimization of analytics tools outside of the gaming domain will be continuously monitored and reported through systematic state-of-the-art reviews and through active participation to relevant conferences and workshop events. The ultimate goal is to integrate advances in the state-of-the-art so as to improve the analytics outcomes and thereby achieve the optimal impact.

Acknowledgments The research leading to these results has received funding from ENVISAGE project that has received funding from the European Union's Horizon 2020 research and innovation programme under grant agreement No 731900.

\section{References}

Clark, A. (1998). Being there: Putting brain, body, and world together again. MIT press.

Drachen, A., Thurau, C., Yannakakis, G., Togelius, J., \& Bauckhage, C. (2013). Game Data Mining. In: Game Analytics - Maximizing the Value of Player Data, Seif El-Nasr, M.; Drachen, A. \& Canossa, A. (Eds.). Springer Publishers [URL: http://www.springer.com/computer/hci/book/978-1-4471-4768-8], pp. 205-253.

Greller, W., \& Drachsler, H. (2012). Translating Learning into Numbers: A Generic Framework for Learning Analytics. Educational technology \& society, 15(3), 42-57.

Lauría, E. J., Moody, E. W., Jayaprakash, S. M., Jonnalagadda, N., \& Baron, J. D. (2013, April). Open academic analytics initiative: initial research findings. In Proceedings of the Third International Conference on Learning Analytics and Knowledge (pp. 150-154). ACM.

Liapis, A., Yannakakis, G. N., \& Togelius, J. (2014, August). Designer modeling for sentient sketchbook. In Computational Intelligence and Games (CIG), 2014 IEEE Conference on (pp. 1-8). IEEE.

Runge, J., Gao, P., Garcin, F., \& Faltings, B. (2014, August). Churn prediction for high-value players in casual social games. In Computational Intelligence and Games (CIG), 2014 IEEE Conference on (pp. 1-8). IEEE.

Shieh, L., Pummer, E., Tsui, J., Tobin, B. \& Hooper, K., (2012). Increasing knowledge of sepsis recognition and management through a mobile educational game. In AAMC Annual Meeting.

Siemens, G., \& Long, P. (2011). Penetrating the Fog: Analytics in Learning and Education. EDUCAUSE review, 46(5), 30.

Siemens, G., \& Long, P. (2011). Penetrating the fog: Analytics in learning and education. EDUCAUSE review, 46(5), 30. 
Sloman, A. (2002). Diagrams in the mind? (pp. 7-28). Springer London.

Tsui, J., Lau, J., \& Shieh, L. S. (2014). SICKO: implementing and using learning analytics and gamification in medical education. Educause; 2014. 